\title{
Estudio de fatiga en mezclas asfálticas recicladas con cemento a través de ensayo en viga cuatro puntos
}

\author{
Mario Alexander Castañeda López \\ William Fedrigo \\ Thaís Radünz Kleinert \\ Estudiantes de Posgrado, Universidad Federal de Río Grande del Sur, Brasil \\ Washington Peres Núñez \\ Jorge Augusto Pereira Ceratti \\ Docentes asociados, Universidad Federal de Río Grande del Sur, Brasil \\ Valeria Cristina de Faria \\ Luis Miguel Gutierrez Klinsky \\ Investigadores, Concesionaria de vías CCR Nova Dutra, Brasil
}

\section{RESUMEN}

El reciclaje profundo de pavimentos flexibles con cemento Portland es una técnica de recuperación estructural y funcional recurrente en la práctica vial Brasilera, ya que la reutilización de capas degradadas de revestimiento asfáltico o RAP (reclaimed asphalt pavement) y de base, permite reducir costos y tiempo de ejecución. Sin embargo, en Brasil no existe un criterio de dimensionamiento de pavimentos que considere este tipo de materiales. En este sentido, es aceptado que la capacidad estructural de capas cementadas es limitada por la deformación de tracción en su base. Con el objetivo de establecer esta relación, a través de modelos de fatiga, fue desarrollado un programa experimental basado en la experiencia australiana, buscando verificar su aplicabilidad a materiales reciclados de origen brasilero. Conforme al protocolo adoptado, el programa consistió de ensayos de flexión estáticos y dinámicos del tipo cuatro puntos en vigas, con edades de cura mayores a 28 días, moldadas en laboratorio usando energía de compactación modificada brasilera. Las mezclas consideradas están constituidas $4 \%$ de cemento Portland, y por brita granular y RAP en proporciones 80:20, 50:50 y 30:70. El comportamiento mecánico fue evaluado a través de la Resistencia a la Tracción a la Flexión (RTF) y el Módulo Flexural Estático y Resiliente (MFE y MFR, respectivamente). Entre otras conclusiones, el comportamiento estático indica que el incremento de RAP reduce el MFE, incrementando la deformación de tracción de ruptura $\left(\varepsilon_{t}\right)$, mientras que la influencia del RAP en la RTF no fue notoria. De otra parte, la relación entre el exponente de daño y el porcentaje de RAP apunta a una dependencia del fenómeno de fatiga y el contenido de material reciclado. En general, puede concluirse que el protocolo australiano puede ser usado como modelo para el desarrollo de un método brasilero para este tipo de mezclas, con algunas modificaciones.

\section{INTRODUCCIÓN}

Al ser el más usado en Brasil, el modo vial ha sido afectado por cuenta del crecimiento económico que durante las últimas décadas se ha experimentado en términos de incremento de tráfico y cargas transportadas, propiciando la ejecución de un mayor número de 
reparaciones y demandando nuevas estructuras que garanticen un adecuando nivel de servicio. En este sentido, los métodos tradicionales de reparación representan altos costes y pasivos ambientales asociados, mientras que la proyección de nuevas estructuras, con mayor capacidad estructural, usualmente lleva a la inclusión de capas asfálticas con mayores espesores que además del incremento de costos, pueden promover otro tipo de patologías asociadas a deformaciones plásticas de la carpeta asfáltica. Así, la tendencia en países con más tecnología en pavimentación está dirigida a la inclusión de capas artificialmente cementadas en conjunto con pavimentos flexibles, apuntando a la utilización de los denominados pavimentos semirrígidos o invertidos.

Ante este panorama, la reutilización de materiales y la reducción de costes, ha permitido que la técnica de reciclaje de pavimentos "in situ" con cemento para su uso como base y subbase se consolide como una alternativa atractiva, ya que de una parte, la disponibilidad de residuo fresado es alta, y de otra, la inclusión de camadas cementadas reduce las solicitaciones sobre la subrasante en lo que respecta al dimensionamiento de la estructura. No obstante, aunque la técnica viene siendo utilizada en Brasil por décadas y se cuentan con normas que abordan aspectos ejecutivos de la misma, equipos, y dosificación de mezclas (DER-PR, 2005; DERSP, 2006; DNIT 167/2013-ES), la consideración de este tipo de materiales a nivel de diseño de pavimentos no se encuentra muy desarrollado.

En este sentido, tradicionalmente en las metodologías de diseño de pavimentos del tipo mecanístico con inclusión de camadas cementadas, el factor que limita los diseños es la RTF y la deformación de tracción en la base de la camada. Es así como métodos de dimensionamiento desarrollados recientemente que consideran materiales reciclados (AUSTROADS, 2004; SANRA, 2010), utilizan relaciones de fatiga en función de esfuerzo o de la deformación de tracción en la fibra inferior de la camada, para estimar un número de ciclos necesarios para su degradación hasta un nivel aceptable según la categoría de la vía, previo un ajuste laboratorio-campo que también depende de tal categoría, de tal forma que satisfaga las exigencias de tráfico definidas en estudios de tránsito.

De esta forma, el presente estudio busca conocer la interacción entre la reducción de la rigidez flexural del material ante cargas cíclicas a través de ensayos de fatiga tipo cuatro puntos con el objetivo de establecer modelos preliminares de fatiga en laboratorio (sin adopción de factores laboratorio-campo), que permitan evaluar la influencia de la presencia de RAP en conjunto con agregados vírgenes para mezclas estabilizadas con cemento Portland.

\section{PROGRAMA EXPERIMENTAL}

La metodología está basada en el Protocolo Australiano AP-T101/08 (AUSTROADS, 2008) y AP-T198-12 (AUSTROADS, 2012), siendo que estos han sido desarrollado inicialmente para materiales cementados sin inclusión de materiales reciclados. Así, criterios relacionados con materiales y mezclas fueron establecidos siguiendo procedimientos del manual alemán 
WIRTGEN (WIRTGEN, 2012). La metodología se divide en definición de materiales, mezclas, ensayos de compactación, y la preparación y ensayo de los cuerpos de prueba, esto es, ensayos estáticos y dinámicos (fatiga).

\subsection{Materiales y mezclas}

Fue definida la utilización de agregado virgen constituido por una mezcla de grava (tamaño 3/8" y 3/4"') y arena, en proporciones que representen la granulometría de un material granular reciclado. Debido a que la granulometría del material granular se ajustó al criterio propuesto, éste finalmente determinó los límites del contenido máximo de RAP a utilizar sin la necesidad de estabilización granulométrica con otros materiales. Así, fueron definidos tres porcentajes de RAP, en masa: 20, 50 y 70. El cemento utilizado es del tipo Portland compuesto, de resistencia intermedia con adición de escoria (CP II 32 E, nomenclatura brasilera) de amplio uso en este tipo de reciclaje de pavimentos, en un porcentaje, en masa, del $4 \%$. Por otro lado, fue realizado ensayo de extracción de ligante por reflujo siguiendo la norma brasilera DAER/RS EL 213/01 (DAER, 2001). Las mezclas son nombradas en función del contenido de cemento y fresado: 4-20, 4-50 y 4-70.

\subsection{Compactación}

Fueron efectuados ensayos de compactación dinámica siguiendo la normativa brasilera (DNIT, 2013), energía modificada.

\subsection{Preparación de cuerpos de prueba}

A partir de los parámetros de compactación definidos, se moldaron siguiendo el protocolo estadounidense NCHRP-789 (NCHRP, 2014) 12 cuerpos de prueba prismáticos para cada mezcla: 3 para Resistencia de Tracción a la Flexión (RTF), y 9 para fatiga. Las dimensiones (100 mm x 100 mm x $400 \mathrm{~mm}$ ) fueron establecidas en función del máximo tamaño nominal de los agregados. La preparación consistió en la mezcla de la brita y el fresado, adición del cemento y agua para alcanzar la humedad óptima y su posterior compactación estática en tres capas iguales por medio del control de altura de la prensa en función de la masa específica aparente seca. Después de desmoldar, dos días después, los cuerpos de prueba son llevados a la cámara húmeda donde son mantenidos a $21 \pm 2{ }^{\circ} \mathrm{C}$ y $90 \%$ de humedad relativa durante un mínimo de 28 días.

\subsection{Resistencia de tracción a la Flexión}

El ensayo RTF así como los cálculos, siguen recomendaciones de la NCHRP (NCHRP, 2014) y JSCE-SF4 (JCI, 1984). Esta última es tradicionalmente tenida como referencia para la estimación de la tenacidad de concreto con adición de fibras, siendo que su consideración obedece al arreglo utilizado para medición de desplazamientos.

El ensayo consiste en la aplicación de una carga estática, constante, de 2,3 kN/min, distribuida en dos apoyos superiores situados en el tercio medio del vano de $300 \mathrm{~mm}$ que ofrecen los apoyos inferiores, como es mostrado en la Figura 2. Tal disposición garantiza 
una ruptura por flexión pura, es decir, sin considerar efectos de esfuerzos cortantes, simulando de una forma más próxima a la realidad el comportamiento en campo de una capa cementada. El desplazamiento ocasionado por la viga es medido con dos LVDTs (Linear Variable Differential Transformer), ubicados uno a cada lado de la viga como es mostrado en la Figura 2. Los datos de desplazamiento son empleados para la estimación de deformaciones de tracción de la base del cuerpo de prueba.

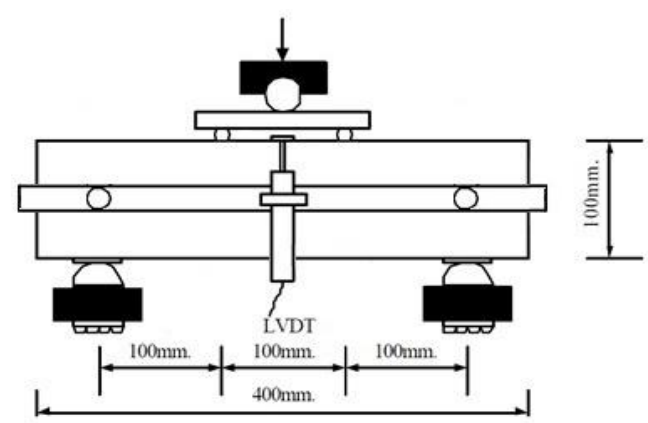

Fig. 1 - Arreglo de ensayo de RTF y de fatiga (adaptado de Suthiwarapirak, Matsumoto y Tetsushi, 2002)

\subsection{Módulo Flexural y fatiga cuatro puntos}

El ensayo de fatiga así como los cálculos, siguen directrices establecidas en los protocolos australianos AP-T101/08 (AUSTROADS, 2008) y AP-T198-12 (AUSTROADS, 2012). Consiste en la aplicación de una carga cíclica de magnitud constante, empleando el mismo arreglo al mostrado en la Figura 2. Son registrados valores de carga y desplazamiento asociado, siendo que la amplitud máxima de los ciclos, en valores absolutos, determina su magnitud neta, como es mostrado en la Figura 2. El promedio de los valores de esfuerzo y deformaciones entre los ciclos 50 a 100, definen la deformación de tracción inicial.

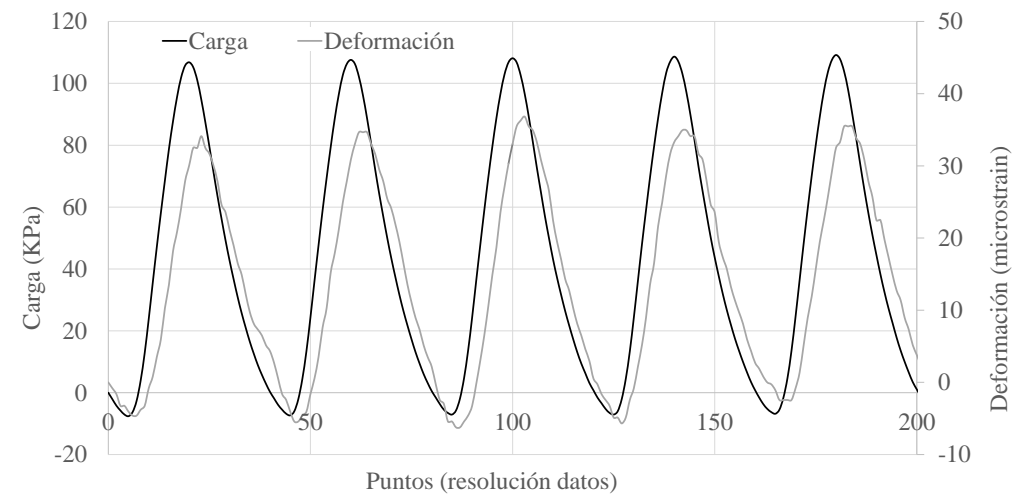

Fig. 2 - Ejemplo de pulso aplicado y deformación (1 s de ensayo)

\subsubsection{Ciclos de carga}

El tipo de onda aplicado es del tipo semiseno (haversine). La frecuencia utilizada fue incrementada a $5 \mathrm{~Hz}$ tanto para módulo como para fatiga, que equivale a una velocidad de tráfico de $30 \mathrm{~km} / \mathrm{h}$ como relata la experiencia brasilera en este tipo de ensayos (Paiva y Oliveira, 2014). 
Respecto a la magnitud de cargas aplicadas, aunque la recomendación australiana indica la estimación del módulo flexural inicial para $40 \%$ de la RTF, resultados iniciales para el tipo de mezclas estudiado mostraron que provoca su degradación prematura, implicando una inadecuada caracterización resiliente del mismo. De esta forma, fue adoptado otro criterio contenido en el mismo protocolo que indica que la respuesta de la viga, en términos de deformación debe ser entre 20 y 30 microstrains $\left(10^{-6} \mathrm{~mm} / \mathrm{mm}\right)$ para módulo y entre 50 y 100 microstrains para fatiga.

\section{RESULTADOS Y ANÁLISIS DE RESULTADOS}

Los resultados son agrupados en términos de caracterización (granulometría, parámetros de compactación), RTF (RTF, MFE y deformación estática en la ruptura) y curvas de fatiga.

\subsection{Caracterización}

Las tres mezclas consideradas se encuadran en los límites granulométricos propuestos. De otra parte, el contenido de ligante extraído del RAP, en peso, fue de 3,84\%. Respecto a los parámetros de compactación, la masa específica aparente seca óptima fue de $2,1 \mathrm{~g} / \mathrm{cm}^{3}$ para las tres mezclas, en cuanto que la humedad óptima varió entre 7,0 \% y 8,3\%.

\subsection{Resistencia de tracción a la Flexión}

Los resultados de los ensayos RTF corresponden al promedio de ensayo de tres cuerpos de prueba. A partir de las curvas esfuerzo deformación son elaborados los gráficos mostrados en la Figura 3 (der.), donde se presenta una reducción de la RTF con el aumento del contenido de fresado aunque, con variabilidad reducida (entre 0,77 y $0,86 \mathrm{MPa}$ ). En cuanto a la deformación en la ruptura, correspondiente a la deformación al $95 \%$ de carga última Figura 3 (izq.), es evidente que la reducción de la rigidez flexural permite desarrollar mayores deformaciones antes de la ruptura, especialmente de $50 \%$ para $70 \%$.
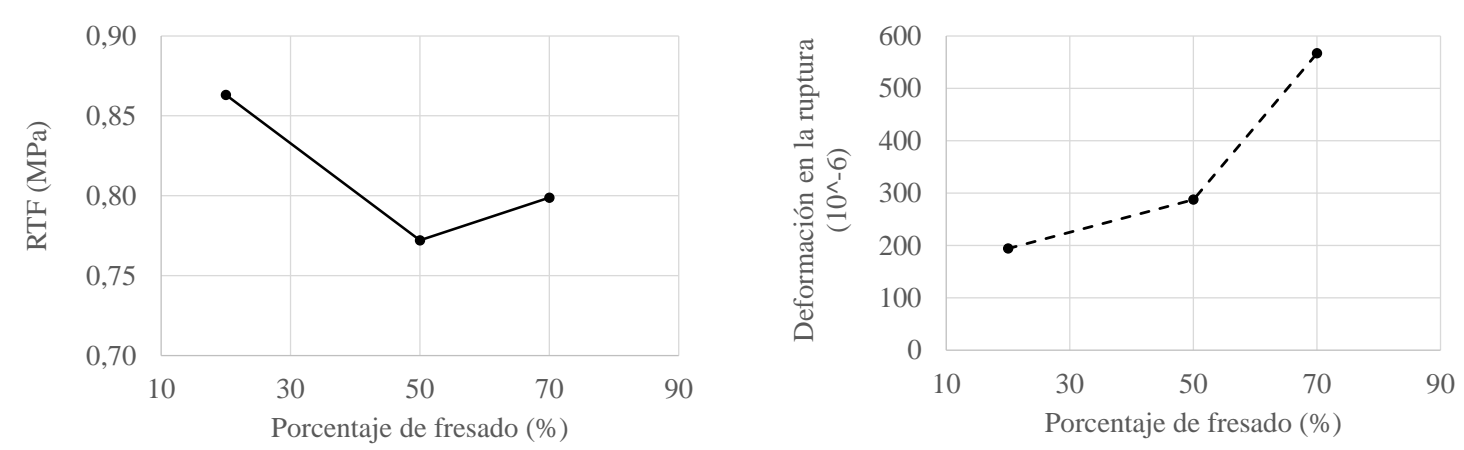

Fig. 3 - Influencia del RAP sobre el compartimiento flexural. Der.: RTF, Izq.: deformación en la ruptura

De forma análoga a las curvas del tipo esfuerzo-deformación, la Figura 4 (der.) representa la variación del MFE (secante) en función de los esfuerzos de tracción normalizados. Así, el efecto de la inclusión del RAP, advertido en términos de deformación en la ruptura se 
extiende a todos los estados de carga. Es destacable la reducción de $20 \%$ para $50 \%$ de RAP en estados de deformación inferiores al $50 \%$ de la RTF. Como referencia es tomado el valor de MFE para el $40 \%$ de la RTF como es mostrado en la Figura 4 (izq.).
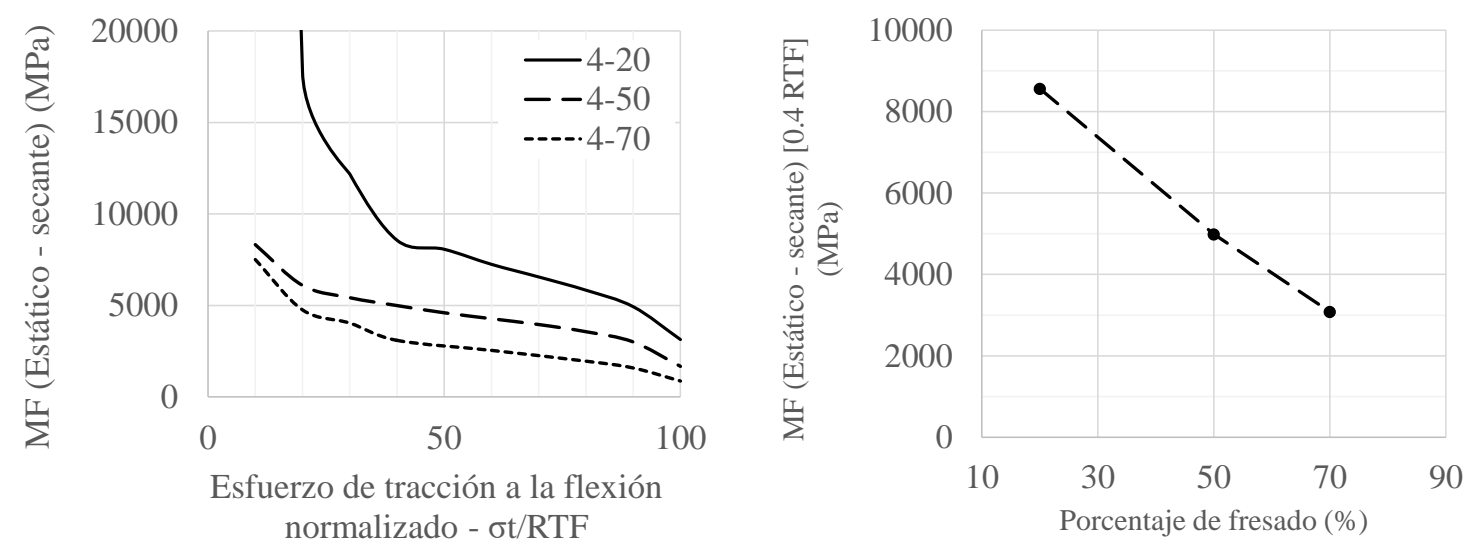

Fig. 4 - Módulo Flexural Estático. Der.: MFE vs. Esfuerzo Normalizado, Izq.: MFE vs. porcentaje de RAP

\subsection{Fatiga}

El comportamiento bajo cargas cíclicas de las tres mezclas estudiadas es evaluado en función de la deformación inicial, $\left(\varepsilon_{i}\right)$ y del número de ciclos necesarios para la ruptura de la viga $(\mathrm{N})$, como es mostrado en la Figura 5. El banco de datos obtenido permitió además, monitorear la degradación del material, como función de la reducción del MFR. El criterio de falla fue la ruptura de la viga, o $\mathrm{N}=10^{6}$ ciclos. Las ecuaciones de fatiga mostradas en la Figura 5, en conjunto con su correspondiente coeficiente de correlación, son expresadas como función de $\mathrm{N}$ para una mejor interpretación, de la forma mostrada en la Ecuación 1, donde $a$ y $b$ son constantes de regresión, siendo que esta última corresponde al LDE (Load Exponent Damage), que de acuerdo a la práctica australiana, define el comportamiento a fatiga de la mezcla.

$$
\varepsilon_{i}=a * N^{-1 / b}
$$

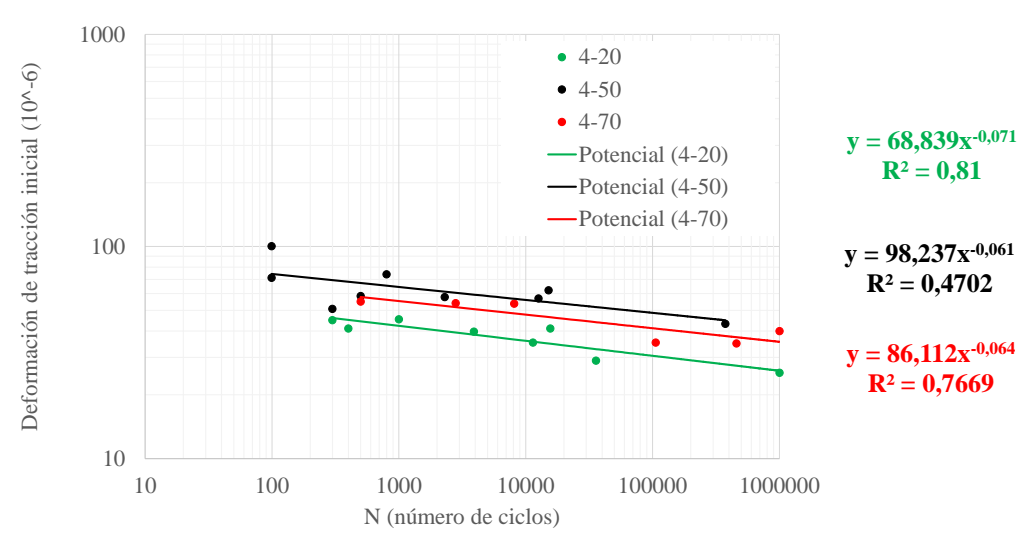

Fig. 5 - Curvas de fatiga en función de la deformación de tracción inicial 
De esta forma, se obtuvieron los siguientes valores de LDE: 14 (4-20), 16 (4-50) y 16 (470). De acuerdo con la experiencia australiana (AUSTROADS, 2004), sin inclusión de materiales reciclados, el valor de LDE es 12, siendo que los resultados obtenidos apuntan a la susceptibilidad del LDE con el aumento del contenido de RAP. Esto indica que, de forma similar a los resultados de los ensayos estáticos, al tornarse menos rígida la mezcla con el incremento de contenido de RAP (en términos de rigidez flexural), se generan mayores deformaciones antes de la propagación de la fractura bajo un estado de carga dinámico.

\section{CONCLUSIONES}

Las siguientes conclusiones pueden ser establecidas a partir de los resultados obtenidos:

- La metodología adoptada mostró ser adaptable para el estudio del comportamiento flexural de los materiales analizados.

- En el caso de ensayos estáticos, el contenido de fresado tiene mayor influencia en términos de deformación que en términos de RTF.

- La influencia del RAP sobre la rigidez flexural (tanto estática como dinámica), es mayor de $20 \%$ para $50 \%$.

- El incremento del valor del LDE con el contenido de RAP como consecuencia de la reducción de la rigidez flexural de cada mezcla limita su uso, al reducir la capacidad estructural de las capas superiores.

- Los valores de LDE obtenidos, próximos a los encontrados en la literatura, y que son fundamentales en términos de dimensionamiento de pavimentos con inclusión de capas cementadas sugiere que este tipo de mezclas puede ser susceptible de ser adaptada al método de dimensionamiento de la AUSTROADS (2004), requiriendo estudios más amplios en este sentido.

- Así, resulta necesario estudios de fatiga para otros contenidos de cemento, y otros tipos de materiales de base usualmente empleados dentro de la práctica brasilera, generando mayores bancos de datos con el fin de asegurar una adecuada representatividad de los mismos como paso previo al desarrollo de un método de dimensionamiento.

\section{AGRADECIMIENTOS}

Los autores agradecen a becarios, técnicos, funcionarios, investigadores y profesores de los laboratorios de la Universidade Federal do Rio Grande do Sul (UFRGS) envueltos en el estudio: Laboratório de Pavimentação (LAPAV), Laboratório de Estruturas y Modelos Estruturais (LEME) y Laboratório de Geotecnologia (LAGEOTEC). A la CAPES y CNPq, entidades financiadoras de las becas de estudios de posgrado e investigación que permitieron el desarrollo de la misma. Se extienden los agradecimientos a las entidades adscritas al proyecto de reciclaje de pavimentos: Tecnopav, Wirtgen, EcoRodovias, Grupo CCR, Instituto de Pesquisas Rodoviárias (IPR - DNIT), Associação Brasileira de Cemento Portland (ABCP) y Agência Nacional de Transportes Terrestres (ANTT). 


\section{REFERENCIAS}

AP-T101/08. AUSTROADS. Australia. Association of Australian and New Zealand road transport and traffic authorities. 2008.

AP-T198-12. AUSTROADS. Australia. Association of Australian and New Zealand road transport and traffic authorities. 2012.

AUSTROADS. (2004). Guide to the Structural Design of Road pavements. Australia.

DAER/RS EL 213/01. DAER. Brasil. Departamento autônomo de estradas de rodagem. 2001.

DER-PR ES-P 33/05. DER. Brasil. Departamento de estradas de rodagem do Estado do Paraná. 2005.

DER-SP ET-DE-P00/035. DER. Brasil. Departamento de estradas de rodagem do Estado de São Paulo. 2006.

DNIT 164/2013-ME. DNIT. Brasil. Departamento nacional de infraestrutura de transportes. 2013.

DNIT 167/2013. DNIT. Brasil. Departamento nacional de infraestrutura de transportes. 2013.

JSCE SF4. Japón. Japan Concrete Institute. 1984.

NCHRP-789. DNIT. Estados Unidos. National Cooperative Highway Research Program. 2014. Report.

PAIVA, C. E. L.; OLIVEIRA, P. C. A. (2014). Impacto da Atividade do CAP e do Percentual de Asfalto Fresado na Resistência a Fadiga de uma Base Reciclada com Cimento. 21o Encontro de Asfalto. Ponencia. Instituto Brasileiro de Petróleo, Gás e Biocombustíveis, Rio de Janeiro, Brasil.

SANRA. (2013). South African Pavement Engineering Manual. South Africa.

SUTHIWARAPIRAK, P; MATSUMOTO, T.; TETSUSHI, K. (2002). Flexural fatigue failure characteristics of an engineered cementitious composite and polymer cement mortars. Japón.

WIRTGEN. (2012). Wirtgen cold recycling technology. Windhagen, Alemania. 